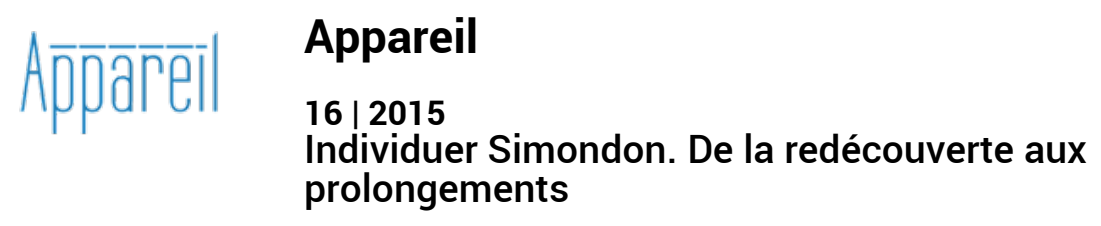

\title{
Simondon et le langage
}

Ludovic Duhem

\section{CpenEdition}

Journals

Édition électronique

URL : http://journals.openedition.org/appareil/2223

DOI : 10.4000/appareil.2223

ISSN : 2101-0714

Éditeur

MSH Paris Nord

Référence électronique

Ludovic Duhem, «Simondon et le langage », Appareil [En ligne], 16 | 2015, mis en ligne le 03 février 2016, consulté le 30 juillet 2020. URL : http://journals.openedition.org/appareil/2223 ; DOI : https:// doi.org/10.4000/appareil.2223

Ce document a été généré automatiquement le 30 juillet 2020.

\section{(c)}

Appareil est mis à disposition selon les termes de la Licence Creative Commons Attribution - Pas d'Utilisation Commerciale - Pas de Modification 4.0 International. 


\title{
Simondon et le langage
}

\author{
Ludovic Duhem
}

« Le discours est l'un des genres des êtres, énonce

l'Étranger.

Privés de cela, qui est la chose la plus importante, nous serions privés de la philosophie ».

Platon, Le Sophiste

\section{Introduction : l'aporie de la question du langage}

1 Malgré l'existence, désormais établie, d'une réception scientifique et internationale de la pensée de Simondon qui s'appuie autant sur l'édition française de l'ensemble de l'œuvre et ses traductions en cours que sur les multiples travaux universitaires qui paraissent partout dans le monde, tout lecteur attentif de son œuvre et de ses commentateurs rencontre une question qui, à force de rester sans réponse, résonne comme une aporie : qu'en est-il du langage pour Simondon?

2 Cette question, simple en apparence, devient en effet aporétique si l'on considère le peu de réflexions que Simondon lui consacre, alors que toutes les conditions semblent réunies pour qu'il pose le problème du langage et en propose une théorie cohérente et originale.

Pour s'en convaincre, il suffirait que l'on convoque :

3 a) tout d'abord les circonstances historiques de l'élaboration de la pensée de Simondon, qui sont celles d'une époque où les théories du langage dominent en philosophie comme dans les sciences humaines (au moins sous la forme du structuralisme linguistique et du logicisme analytique) ;

b) les enjeux philosophiques de la théorie de l'individuation, laquelle repose sur une critique fondamentale du substantialisme qui implique non seulement le dépassement des oppositions ontologiques, épistémologiques et logiques, mais aussi une profonde réforme méthodologique et notionnelle qui révoque la pertinence du concept (dans son sens classique) pour penser la réalité ; 
5 c) le sens politique de la formation d'une culture complète, universelle et régulatrice pour sortir la société de l'aliénation à l'époque des réseaux informationnels. Cette culture étant fondée sur un "nouvel encyclopédisme " qui atteste et renforce le passage d'une civilisation du langage à une civilisation de l'image.

Si ces trois motifs légitiment donc une investigation du sens de l'absence de théorie du langage chez Simondon, deux hypothèses peuvent cependant expliquer cette «absence »:

6 la première hypothèse est que Simondon n'avait pas besoin de poser le problème du langage, parce que :

7 1. la philosophie génétique et relationnelle qu'il propose est incompatible avec la tradition substantialiste qui définit le logos comme origine de la vérité, comme raison de la phusis, comme structure de toute pensée, ce qui contrevient à toute connaissance de l'individuation en imposant le primat de l'être sur le devenir, l'opposition du sujet et de l'objet, l'hégémonie du principe d'identité et de la logique du tiers-exclu (L'individuation à la lumière des notions des forme et d'information);

8 2. la réflexivité propre à la pensée philosophique est irréductible à la discursivité en général, c'est-à-dire à la logique comme à la langue, puisque les deux conditions constitutives de la « vie réflexive » sont la « vie organique » et la « vie technique » (Note complémentaire) ;

9 3. la philosophie, en tant que mode réflexif de relation de l'homme au monde, n'est ni la première ni la seule forme de pensée, puisqu'il existe une pensée religieuse, une pensée technique et une pensée esthétique qui lui sont antérieures et avec lesquelles la pensée philosophique forme un système polyphasé (Du mode d'existence des objets techniques).

10 La seconde hypothèse est que Simondon a envisagé autrement le problème du langage en posant un problème à la fois plus fondamental et plus large : celui du rapport entre signification et information dans la communication (Communication et information). D'une part, ce problème est plus fondamental parce qu'il nécessite de replacer le langage dans le système des phases de la genèse de l'individu et dans la complexité des niveaux de sa relation à lui-même et au monde, ce qui fait apparaître le langage comme une réalité relative, localisée et tardive ; d'autre part, ce problème est plus large que le problème du langage, parce qu'il traverse autant le vivant non-humain que le vivant humain, les objets esthétiques et sacrés que les objets techniques, selon des modalités que tout paradigme linguistique tendrait à appauvrir.

11 En d'autres termes, en écartant le langage comme condition première de la pensée et $\mathrm{du}$ sens, la première hypothèse ferait de la philosophie de Simondon une critique fondamentale du logocentrisme. Cela le placerait immédiatement dans la lignée des critiques nietzschéenne et bergsonienne du langage, dont il hérite directement, et en parallèle de celle proposée par Heidegger, avec lequel il n'est jamais vraiment entré en dialogue. Il faudrait alors expliciter ce que peut signifier "penser sans partir du langage » selon sa propre philosophie et en quoi elle pourrait être une alternative à la «parole» heideggerienne. Quant à la seconde hypothèse, elle affirmerait que le problème du langage est en vérité un faux problème, c'est-à-dire un problème mal posé, en tant qu'il dépend d'une représentation anthropocentrique et réductionniste de la pensée qui entrave la compréhension des fonctions respectives de l'information et de la signification selon les différents niveaux de communication et la diversité des êtres vivants capables de penser. 
12 L'ambition de cet article ne sera pas d'apporter une vérification systématique et approfondie de ces deux hypothèses, mais de proposer un premier « état des lieux » de la question du langage dans l'œuvre de Simondon qui puisse intégrer la critique du logocentrisme et la critique de l'anthropocentrisme pour les préciser. Trois aspects seront plus particulièrement examinés :

13 1) la critique du langage compris comme condition de la connaissance et de la pensée ;

14 2) les tensions internes au rapport entre technique et langage ;

15 3) les éléments d'une théorie communicationnelle du langage.

Il s'agira donc de poser ici le problème du langage dans la philosophie de Simondon sans prétendre éviter l'aporie énoncée; au contraire, il faudra plutôt endurer cette dernière, afin de mieux la délimiter et d'en comprendre plus précisément les effets herméneutiques.

\section{Critique du langage et théorie du sujet}

16 C'est dans le cadre de sa pensée de l'individuation que Simondon propose une critique du langage. Mais cette critique, sans être implicite, n'est ni directe ni systématique. Elle relève avant tout du geste initial de renversement ontologique du substantialisme et de ses conséquences épistémologiques et logiques.

Dès l'« Introduction » de sa Thèse principale, Simondon montre ainsi que les deux voies traditionnelles pour penser l'«être comme individu», le substantialisme et l'hylémorphisme, ont un présupposé commun: l'existence d'un "principe d'individuation antérieur à l'individuation elle-même, susceptible de l'expliquer, de la produire, de la conduire ${ }^{1}$.» Or, un tel présupposé empêche toute connaissance de l'individuation, car non seulement un "privilège ontologique " est accordé à «l'individu en tant qu'individu constitué », mais la recherche d'un principe force à opérer une "genèse à rebours" pour retrouver le terme premier, responsable de l'existence et des caractéristiques de l'individu à expliquer. Cette recherche du terme premier, analogue à la recherche du substrat des prédicables, implique nécessairement de négliger l'opération d'individuation, voire de sauter par-dessus pour rejoindre le principe qui est au commencement et au commandement de la réalité. Mais cette recherche implique surtout d'expliquer l'individuation de l'individu avec un terme premier, c'est-à-dire avec ce qui est «déjà un individu ou quelque chose d'individualisable ». L'opération d'individuation elle-même reste donc obscure et ne peut faire l'objet d'une connaissance réelle. C'est pour éviter un tel manque que Simondon propose un renversement épistémologique consistant à «connaître l'individu à travers l'individuation plutôt que l'individuation à partir de l'individu ${ }^{2}$ ». Par ce renversement, l'opération d'individuation devient primordiale, elle n'est plus dépendante d'un principe antérieur mais elle est à elle-même son principe; corrélativement, l'individu n'est plus une réalité absolue mais une phase dans un processus qui se déroule au sein d'un système.

18 Pour penser l'individuation, il est donc impossible d'avoir recours au modèle de la substance et à la logique qui lui correspond. La logique substantialiste repose en effet sur l'idée que la substance est ce qui consiste en son unité et subsiste en son identité, c'est-à-dire ce qui obéit sans exception au principe du tiers-exclu (tout être est soit $\mathrm{A}$ soit non-A et il ne peut exister de tiers terme, entre $\mathrm{A}$ et non-A, qui soit à la fois $\mathrm{A}$ et 
non-A ou ni A ni non-A) en vue d'une subsomption des individus sous l'universel. Or, si l'on cherche comme Simondon à connaître l'individu à travers l'individuation, l'unité et l'identité ne sont plus des catégories pertinentes. D'une part, l'unité renvoie à l'individu, à l'être individué, fermé sur lui-même et consistant en lui-même, et non pas à l'être en train de s'individuer ni à l'être préindividuel, qui est « plus qu'unité » dans la mesure où il recèle des potentiels que l'individu actualise sans les épuiser au cours de son individuation. D'autre part, l'identité renvoie à l'idée que le devenir de l'être ne peut être autre chose qu'un cadre sans conséquence sur ce qu'il en est de l'être pour cet être, demeurant ce qu'il est selon un état parfaitement stable où aucune transformation n'est possible; alors que la connaissance de l'individuation nécessite au contraire de penser le devenir comme « dimension » de l'être, c'est-à-dire comme ce qui transforme l'être préindividuel en résolvant son incompatibilité initiale riche en potentiels. Il en résulte que "l'unité et l'identité ne s'appliquent qu'à une des phases de l'être », c'est-àdire à la phase où l'individu est apparu, où l'être s'est individué, et non pas à l'opération d'individuation à travers laquelle il s'agit de (le) connaître.

19 Tout recours au concept, pris en son sens classique, est par conséquent inadéquat pour résoudre le problème de la connaissance ${ }^{3}$. Du point de vue de la théorie de l'individuation, un concept n'est en effet "ni a priori ni a posteriori, mais a praesenti ", car il provient d'une opération psychique qui ne se réalise pas de manière abstractive à partir de sensations constituant une matière donnée a posteriori pour les formes a priori de la sensibilité. La formation d'un concept est au contraire une opération d' individuation, c'est-à-dire la résolution d'une problématique qui s'effectue a praesenti selon les phases de l'opération de connaissance, selon les niveaux de l'être connaissant et en incorporant tous les éléments de la situation concrète. Or, conserver la distinction entre a priori et a posteriori proposée par Kant, c'est imposer, selon Simondon, un «retentissement du schème hylémorphique » dans la théorie de la connaissance qui entrave la connaissance de l'individuation par une double illusion quant à la relation du sujet connaissant à l'objet connu. D'une part, il y a l'illusion des formes a priori, qui ne sont pas réellement «a priori » puisqu'elles sont issues d'une première résolution de la problématique produite par l'orientation de l'être vivant dans un monde polarisé, c'està-dire qu'elles servent de structures pour un système préindividuel qui existe et s'individue avant toute constitution d'un objet et d'un sujet. L'illusion des formes a priori s'explique plus précisément par «la préexistence, dans le système préindividuel, de conditions de totalité, dont la dimension est supérieure à celle de l'individu en voie d'ontogenèse ", ce qui peut laisser croire qu'il existe pour l'individu un cadre préalable à toute connaissance possible, alors qu'il s'agit du terme extrême d'ordre supérieur du système dans lequel une problématique apparaît. D'autre part, l'illusion de l'a posteriori participe de la même logique en imposant l'idée que la sensation apporte la matière nécessaire à la connaissance, se constituant comme un donné a posteriori pour les formes a priori, alors qu'il s'agit d'une opération progressive et antérieure à la constitution des cadres spatio-temporels, lesquels apparaitront plus tard avec la perception - cette dernière étant elle-même une opération d'individuation postérieure à la sensation qui produit une compatibilité entre l'être sujet et le milieu. L'illusion de l' a posteriori provient donc de l'existence réciproque "d'une réalité dont l'ordre de grandeur, quant aux modifications spatio-temporelles, est inférieur à celui de l'individu ${ }^{4} »$, ce qui peut laisser croire qu'il existe un donné a posteriori et extérieur à l'individu alors qu'il s'agit d'un terme extrême complémentaire et d'ordre de grandeur inférieur au sein du même système. En ce sens, seul un concept «a praesenti » permet 
de réaliser « une communication informative et interactive entre ce qui est plus grand que l'individu et ce qui est plus petit que lui ${ }^{5}$ » et résoudre la problématique de la connaissance de l'individuation en évitant d'avoir recours à une abstraction réalisée à partir de termes donnés. Autrement dit, il s'agit de se replacer dans la situation réelle où une problématique apparaît, dans le cours du processus et au centre de la relation de connaissance, sans chercher à reconstituer des essences ni à subsumer les individus sous l'universel selon les catégories de genre et d'espèce.

On pourrait donc dire que Simondon considère, de manière analogue à Bergson, que le langage opère une coupe statique dans le mouvement des choses et que les concepts produits par l'intelligence réalisent à la fois une opération de généralisation et d'abstraction qui empêchent une compréhension concrète et complète de la réalité. L'idée de Simondon que les concepts sont "a praesenti» répond ainsi aux «représentations souples, mobiles, presque fluides» exigées par Bergson ${ }^{6}$, selon l'ambition commune aux deux penseurs d' ' accomplir la genèse de la pensée en même temps que s'accomplit la genèse de l'objet ${ }^{7}$ ». L'originalité de Simondon tient cependant dans sa méthode transductive qui, certes, est aussi « intuition » au sens de Bergson, mais qui propose un "paradigmatisme analogique » permettant de penser la singularité de chaque individuation et de comprendre la complexification de la relation des différents régimes d'individuation (physique, biologique et transindividuel) selon des rapports opératoires valides.

21 À proprement parler, il n'est donc pas question pour Simondon de «dépasser » la logique du concept comme le voulait Bergson, mais de proposer une théorie ontogénétique "antérieure à toute logique » et qui devrait "pluraliser la logique » selon les types d'individuation rencontrés, comme le voulait plutôt Bachelard ${ }^{8}$. Toute saisie de la réalité est donc une analogie entre deux opérations, analogie qui ne vise jamais l'universel abstrait mais la résolution d'une problématique réelle en instaurant un mode de communication entre ces deux opérations :

L'individuation $\mathrm{du}$ réel extérieur au sujet est saisie par le sujet grâce à l'individuation analogique de la connaissance dans le sujet; mais c'est par l'individuation de la connaissance et non par la connaissance seule que l'individuation des êtres non sujets est saisie. Les êtres peuvent être connus par la connaissance du sujet, mais l'individuation des êtres ne peut être saisie que par l'individuation de la connaissance du sujet ${ }^{9}$.

Toute connaissance de l'individuation est ainsi une individuation de la connaissance, ce qui renverse tous les principes du logocentrisme de la tradition substantialiste et appelle à " penser au milieu », selon la belle formule de Barthélémy ${ }^{10}$.

23 À partir de ce renversement de la tradition substantialiste et du modèle logique de la prédication, Simondon va proposer une autre critique du langage. Cette critique est plus explicite, elle apparaît lorsqu'il aborde le régime d'individuation psychique et collective ou «transindividuel », à propos duquel est justement formulée la théorie du sujet. Pour résoudre sa problématique psychique et accomplir vraiment son être-« sujet ", l'individu doit en effet participer au collectif, ce qu'il fait à travers la découverte de « significations » qui ne sont pas issues de l'interlocution effective ou de la virtualité dialogique du langage, mais de la "présence » d'autrui. Cette présence n'est pas la simple existence hic et nunc d'un autre être à l'extérieur de soi ; elle n'est pas non plus l'affirmation d'une identité singulière ou la reconnaissance d'une appartenance commune à un genre ou à une espèce ; elle est déjà synonyme d'une nouvelle individuation qui se superpose à la première et la déborde. Cette seconde 
individuation peut s'opérer non seulement parce qu'une attente de complétude existe, mais surtout parce que la présence d'autrui polarise une réserve disponible de l'être individué, qui reste non encore structurée malgré la première individuation. La rémanence de cette "charge de nature » - issue de la phase préindividuelle - lors de la phase transindividuelle est précisément ce qui fait apparaître les significations. Plutôt qu'un rapport entre des identités individuées à travers des structures linguistiques données, une "correspondance» des individus est ainsi requise pour qu'une signification puisse apparaître, c'est-à-dire que se produise une réponse réciproque à la présence de l'autre par la mise en relation des relations des individus à leur charge de nature préindividuelle - qui est non seulement pré-linguistique mais aussi pré-vitale. Il est par conséquent impossible d'assigner à la signification la position exclusive d'un a priori ou d'un a posteriori, puisqu'elle est a posteriori dans la mesure où une seconde individuation est nécessaire pour qu'elle apparaisse, et cette apparition nécessite pour exister un a priori réel qui est la relation du sujet à sa charge de nature préindividuelle. C'est pourquoi on peut dire que la signification est paradoxale, en ce sens qu'elle est la «correspondance des a priori » dans «l'individuation a posteriori» elle est autant découverte que produite.

Partant, l'idée classique selon laquelle le langage précède les significations est alors clairement réfutée par Simondon :

[...] « il est absolument insuffisant de dire que c'est le langage qui permet à l'homme d'accéder aux significations; s'il n'y avait pas de significations pour soutenir le langage, il n'y aurait pas le langage; ce n'est pas le langage qui produit la signification; il est seulement ce qui véhicule entre les sujets une information qui, pour devenir significative, a besoin de rencontrer cet apeiron associé à l'individualité définie dans le sujet; le langage est instrument d'expression, véhicule d'information, mais non créateur de significations. La signification est un rapport d'êtres, non une pure expression; la signification est relationnelle, collective, transindividuelle, et ne peut être fournie par la rencontre de l'expression et du sujet. On peut dire ce qu'est l'information à partir de la signification, mais non la signification à partir de l'information ${ }^{11}$.

La position de Simondon est ici sans équivoque : le langage ne précède, ne produit ni ne donne accès aux significations, ce sont les significations qui soutiennent le langage. Le langage assure simplement les fonctions de véhicule d'information et d'instrument de l'expression, il n'est en rien ce par quoi les sujets se rencontrent comme "sujets", c'est-à-dire comme «termes » d'une relation possible. Le véritable idiome du sujet, c'est la relation transindividuelle par laquelle une information peut devenir significative. Pour être reçue, toute information nécessite une individuation qui s'opère au sein du sujet et selon laquelle un collectif se forme avec l'être qui a émis l'information. En d'autres termes, «découvrir la signification du message provenant d'un être ou de plusieurs êtres ", ce n'est pas établir un rapport logique entre signe et référence selon les règles du langage - qu'il soit naturel ou formel -, « c'est former un collectif avec eux, c'est s'individuer de l'individuation de groupe avec eux ${ }^{12} »$. Le langage apparaît alors comme une réalité secondaire pour le sujet, c'est-à-dire comme ce qui vient après l'individuation du sujet et ce qui vient en plus des significations non linguistiques qui apparaissent entre les sujets.

De cette secondarité du langage, il ne faudrait pas conclure que Simondon niât la spécificité du langage humain ; il dit seulement, et c'est beaucoup, que le langage n'est pas au principe de la signification et n'est pas non plus ce qui constitue le sujet en tant que sujet. En renversant ainsi le rapport entre sujet et langage, entre langage et 
signification, Simondon relativise radicalement la place habituellement accordée au langage, au point d'apporter ici une critique fondamentale du logocentrisme retentissant sur l'anthropocentrisme qui le soutient et le légitime. Partant, il est donc faux d'affirmer que l'homme est l'être de la parole, celui qui dit, en tant que sujet, ce qu'il en est de l'être en tant qu'être selon la signification que le langage lui apporte.

\section{Les tensions internes à la pensée simondonienne du rapport langage-technique}

Dans son ouvrage $\mathrm{Du}$ mode d'existence des objets techniques, Simondon propose une philosophie des techniques qui prolonge la théorie de l'individuation élaborée dans sa Thèse principale. Si ce prolongement reprend les principes d'une pensée génétique et relationnelle appliquée à la technique, il implique surtout un "nouvel encyclopédisme » capable de dépasser l'alternative entre humanisme et technicisme, en vue de produire « une prise de conscience du sens des objets techniques » contre le « ressentiment » culturel dominant, source première de l'aliénation contemporaine.

Lorsqu'il s'agit d'examiner les conditions de ce nouvel encyclopédisme, Simondon propose une analyse des trois époques de l'encyclopédisme (éthique, technique et technologique). Après la Renaissance, qui avait produit un premier effort pour « refuser la limitation arbitraire de la pensée et du savoir » en revenant aux textes originaux de l'Antiquité, et avant l'époque contemporaine où domine l'information encodée par les machines, l'époque des Lumières avait libéré la technique par la science et proposé un symbolisme visuel adéquat aux objets techniques. Selon Simondon, l'Encyclopédie est ainsi le lieu où s'invente un nouveau rapport entre langage et image, ou plutôt elle est précisément le lieu du renversement de la domination du langage sur l'image. Un tel renversement s'explique par le fait que :

$[. .$.$] « la technologie exige un moyen d'expression autre que l'expression orale, qui$ utilise des concepts déjà connus, et qui peut transmettre des émotions, mais assez difficilement exprimer des schèmes de mouvement ou des structures matérielles précises; le symbolisme adéquat à l'opération technique est le symbolisme visuel, avec son riche jeu de formes et de proportions. La civilisation du mot laisse place à celle de l'image. Or, la civilisation du mot est par nature même plus exclusive que celle de l'image, car l'image est par nature universelle, ne nécessitant pas un code préalable de significations ${ }^{13}$.

Inadéquat à l'individuation, le langage est aussi inadéquat à la réalité technique, parce qu'il est incapable d'exprimer les schèmes de mouvement et les structures matérielles des objets techniques, c'est-à-dire de donner accès au fonctionnement qui définit leur essence propre comme leur appartenance à une lignée évolutive. Cette incapacité tient d'une part à l'utilisation de concepts donnés qui fixent l'objet et ses composants dans des limites qui le séparent de son milieu et de sa genèse, alors que le symbolisme visuel traduit par un «jeu de formes et de proportions " un mouvement, une structure et possiblement une genèse et une évolution; d'autre part, cette incapacité du langage à exprimer la réalité technique tient à la tendance à la fermeture, au chiffrage, voire aux formules secrètes d'une communauté d'initiés (lorsque le langage est un langage de métier), alors que le symbolisme visuel est immédiat dans la perception et réellement universel dans la connaissance. Ainsi :

[...] «toute expression verbale tend à devenir initiatique; elle se spécialise en aboutissant à une sorte de langage chiffré, dont les anciens jargons corporatifs sont 
un exemple net. Il faut faire partie d'un groupe fermé pour comprendre le langage oral ou écrit; il suffit de percevoir pour comprendre l'expression schématique. C'est avec le schéma que l'encyclopédisme technique prend tout son sens et son pouvoir de diffusion, en devenant véritablement universel ${ }^{14}$.

Tout l'enjeu de la critique simondonienne du langage tient donc ici à la limitation qu'il impose à la connaissance, cette limitation aussi bien sensible que cognitive se redoublant d'une fausse universalité qui entrave l'avènement d'un encyclopédisme réel. Contrairement à l'image ${ }^{15}$, le langage propose, en effet, une fausse universalité dans la mesure où il laisse croire à un accès libre et sans restriction à la connaissance, alors que cet accès est systématiquement sanctionné par la «culture constituée » et le plus souvent réservé à un groupe social qui la représente. À la Renaissance, lors de la première phase éthique de l'encyclopédisme, la diffusion des textes par l'imprimerie apportait un premier élargissement, mais c'était avant tout un message oral qui était véhiculé par les signes visuels, leur signification nécessitant un détour par l'institution sociale du langage pour être accessible. À cette époque où les techniques étaient peu formalisées et difficilement formalisables à cause du développement restreint des sciences, il aurait fallu maitriser toutes les langues vivantes pour que l'encyclopédisme se réalise vraiment. Cela, même une langue savante ne pouvait réaliser, une telle langue restant l'apanage des seuls érudits, interdisant ainsi une universalité de fait, c'est-àdire un exotérisme concret sans initiation ni savoir préalable. C'est pourquoi, même si l'imprimerie a ouvert la voie à une universalité technologique en rendant le symbolisme visuel accessible au plus grand nombre (y compris aux illettrés), il a fallu attendre la fin du XVII ${ }^{\mathrm{e}}$ siècle et surtout la publication de l'Encyclopédie pour que ce symbolisme soit dégagé de «toute volonté d'expression allégorique retournant à une expression orale ${ }^{16} "$, notamment par l'usage précis de la science rationnelle (comme la géométrie) au lieu des références littéraires de la tradition (comme dans les armoiries parlantes).

31 Autrement dit, passer par "l'institution sociale qu'est le langage » signifie d'effectuer un « détour » qui empêche l'universalité technologique. Si la deuxième phase technique de l'encyclopédisme à l'époque des Lumières a ouvert la voie à un schématisme visuel plus adéquat à la réalité technique, obtenu grâce à la libération de la science de l'éthique, celui-ci est encore incomplet : parce que la formalisation des techniques n'est pas totalement réalisée; parce que les corporations maintiennent une fermeture par le langage de métier et les secrets de fabrication; parce que les gravures de l'Encyclopédie contiennent encore des références littéraires et des ornements non fonctionnels. C'est donc une véritable réforme culturelle qu'il faut opérer pour que cette universalité soit effective, complète et non illusoire. Cette réforme culturelle passe par un "nouvel encyclopédisme » fondé sur l'information, qui est plus universelle encore que l'image. Or, malgré les promesses de la cybernétique comme inter-science fondée sur le schème technologique de l'information, et malgré les nouveaux systèmes de production et de diffusion de l'« information figurée » (cinéma et télévision), ce nouvel encyclopédisme attend encore "ses modes d'expression universelle». Selon Simondon, ce retard vient essentiellement du primat culturel accordé à l'expression orale sur l'expression spatiale, qui maintient à l'époque contemporaine le symbolisme visuel à un niveau inférieur d'intérêt et de réflexion alors qu'il pourrait concrétiser l'effort encyclopédique mené depuis la Renaissance. Mais ce retard vient aussi des techniques de production et de diffusion de l'image qui sont pourtant des techniques spatiales: d'un côté, constate Simondon, le cinéma privilégie par nature le mouvement 
dramatique à "la forme intelligible et stable » de la "graphie du simultané », l'image restant soumise au rythme hypnotique propice à la "participation esthétique » et à l'expression des émotions, ce qui fait qu'elle reste pensée comme un «mot » ou une "phrase" et non pas comme un "objet contenant une structure à analyser par l'activité de l'être individuel "; de l'autre côté, la télévision, copie le cinéma dans sa dynamique expressive, nécessite une grande quantité d'information à analyser et à transmettre « sans commune mesure [avec] la quantité d'information significative pour le sujet ${ }^{17}$ ", et reste une technique surtout dédiée à l'asservissement ludique du public au lieu de donner naissance à un nouveau symbolisme universel, ce dont elle est pourtant intrinsèquement capable.

Quant à la cybernétique, il est vrai qu'elle ouvre la voie à un symbolisme universel capable de produire une synergie entre l'homme et la machine, dans la mesure où la théorie de l'information qui lui sert de fondement rend possible un «langage commun » plus universel que le langage naturel. La cybernétique permet en cela de découvrir un codage qui réalise une convertibilité entre l'homme et la machine, qui est certes partielle et encore relativement abstraite, mais qui autorise un couplage qui donne sens à la machine et libère l'homme de son aliénation, sans passer par le prestige du langage ni le filtre de la culture constituée. Mais la cybernétique demeure seulement préparatoire à l'encyclopédisme universel, parce qu'elle reste une pensée fondée sur un technicisme abstrait qui a une vision réductionniste de l'information et cherche à résoudre les problèmes sociaux par l'homéostasie alors qu'il faut penser les conditions d'une société «métastable». C'est en ce sens que Simondon propose une " cybernétique universelle» ou " allagmatique ${ }^{18}$ " qui vise à corriger la cybernétique pour répondre aux enjeux d'un nouvel encyclopédisme où l'information est régulatrice et la culture réellement universelle.

La seconde réflexion sur la relation entre langage et technique chez Simondon apparaitt sous la forme d'une tension entre la théorie des phases de la culture développée par la Troisième partie de la Thèse complémentaire et la théorie analogue proposée dans le texte sur les «Limites du progrès humain » (1959). Cette tension naît de l'absence du langage dans le premier texte et de sa réintroduction dans le second sans que les deux théories soient complémentaires.

Si l'on considère l'analyse des différentes phases du rapport de l'homme au monde que propose le premier texte, aucune de ces phases ne fait l'objet d'une réflexion sur le langage. En effet, dans la phase magique, ni l'incantation aux forces de la nature, ni la légende des événements et des êtres d'exception, ni même les toponymes qui concrétisent la singularité des lieux remarquables ne sont analysés ou seulement abordés. Après le premier déphasage, lorsque s'opposent technique et religion, la technique n'est pas envisagée dans son rapport au langage, alors qu'il avait été question auparavant des termes techniques, des formules secrètes et de l'éducation sans parler du langage en tant que technique: technique du corps, technique de la grammaire, technique de l'écriture. La religion, même à travers la théologie et le dogme, à la fois comme interprétation de la parole divine et comme norme d'action morale, n'est pas non plus mise en relation avec le langage. La pensée esthétique, premier point neutre dans l'enchaînement des phases, n'offre elle aussi que peu de prise au langage : tout au plus le langage est-il rapidement évoqué une première fois comme ce qui « entretient la capacité de penser, sans être la pensée ${ }^{19}$ ", et une seconde fois comme une réalité esthétique restant attachée au corps en prenant la forme d'une 
"modulation de la voix » ou d'une "tournure du discours ${ }^{20}$ " sans que la poésie soit interrogée, y compris lorsque la tragédie est convoquée ${ }^{21}$. La pensée philosophique, au point neutre du deuxième déphasage entre la science et l'éthique, n'est pas présentée comme une pensée discursive, et la réforme qu'elle doit apporter à la culture par l'élaboration d'une "technologie réflexive" qui donne sens aux objets techniques nécessite une nouvelle théorie de la connaissance, et non pas une nouvelle théorie du langage. Tout cela ne montre pas une négligence coupable de la part de Simondon, mais la volonté d'expliquer la genèse de la technicité et le sens de la technique autrement que par la culture littéraire dominante et par le langage comme mode privilégié du rapport de l'homme au monde. La théorie des phases de la culture est donc parfaitement cohérente avec la théorie de l'individuation élaborée dans la Thèse principale.

Dans les «Limites du progrès humain ${ }^{22}$ ", Simondon va pourtant proposer une théorie des phases du progrès humain où le langage est positionné par rapport à la technique et à la religion. Cette intégration du langage dans l'analyse est en partie motivée par la réponse critique que Simondon adresse à un article de Raymond Ruyer lui-même intitulé «Limites du progrès humain ». Dans cet article, l'auteur compare le progrès de la technique scientifique à celui langage, qui finit par s'autolimiter au cours de son évolution ${ }^{23}$. Simondon considère que cette "identification » entre progrès du langage et progrès technique est "réductrice », tout comme l'est celle qui assimile le progrès technique et le progrès humain. Il n'est toutefois pas question pour Simondon de nier qu'une « inhibition interne " puisse exister à l'intérieur d'une concrétisation technique et qu'elle puisse provoquer une autolimitation prenant la forme d'une courbe sigmoïde analogue à celle du langage. Seulement, il existe une saturation progressive du système que forment l'homme et le monde, système qui est d'abord celui du langage puis qui s'engage plus tard dans le développement des techniques, ce dernier système pouvant se saturer à son tour pour laisser place à une autre forme de progrès.

Quoi qu'il en soit, si chaque phase peut s'entendre selon cette évolution par saturation limitative, chacune est aussi reprise et chevauchement. En effet, comme l'explique Simondon, après la saturation du langage des civilisations classiques, la Renaissance, la Réforme et les philosophies éthico-religieuses ont à chaque fois recherché l'esprit du développement du langage pour alimenter l'esprit du développement religieux et technique. Si donc progrès il y a, ce n'est pas selon un principe de saturation absolue ni selon une successivité stricte de concrétisations isolées (langage, technique, religion), mais ce n'est pas non plus selon une évolution déterminée par la constance du recours au langage ${ }^{24}$; en fait, le progrès s'opère selon une augmentation de la résonance interne du système formé par l'homme et ses concrétisations objectives à mesure que ce système passe du cycle autolimité «homme-langage » au cycle " homme-religion » et finalement au cycle « homme-technique ».

Le langage, comme phase primitive et recours préparatoire aux autres phases, n'est donc pas un véritable analogue de la pensée magique telle qu'elle était thématisée par la théorie des phases de sa Thèse complémentaire, car il n'a pas la même nature ni le même statut. Ni la technique ni la religion ne sont à proprement parler des phases issues d'un déphasage du langage, et il n'existe pas, dans «Limites du progrès humain ", de point neutre entre la technique et la religion qui pourrait jouer le rôle de convergence. Sur ce point, les deux théories ne sont ni substituables ni tout à fait complémentaires, elles sont même en partie incompatibles dans leur structure. Quoi 
qu'il en soit, l'évolution propre au langage est la fermeture, lorsqu'il devient par exemple « affaire de grammairiens ou de logiciens formalistes recherchant la rectitude étymologique des dénominations " à la fin de l'Antiquité classique, via un jargon corporatif et ésotérique «sans pouvoir d'expansion constructive ». Mais cette tendance à la fermeture n'est pas pour Simondon la seule raison de refuser d'accorder une universalité réelle au langage, c'est aussi et surtout " parce qu'une grammaire ou une logique formelle ne reflètent pas l'homme, ou tout au moins ne reflètent de l'homme qu'une part minime, et qui ne peut être dilatée ${ }^{25}$ ». Seul l'homme complet, l'homme qui est et qui produit, est en vérité le « moteur » et le " promoteur » de la concrétisation, et non pas l'homme du langage, qui est celui de l'humanisme étroit et pseudo-universel.

Le paradoxe alors énoncé par Simondon est que l'universalité n'augmente pas avec la formalisation et l'abstraction, mais par «décentration " progressive. Le langage est ainsi moins universel que la religion, car la celle-ci concerne en l'homme une réalité plus primitive, moins localisée, plus naturelle, plus implicite. Mais la religion tend aussi à devenir théologie comme le langage tend à devenir grammaire. La technique est quant à elle " encore plus primitive que la religion", car elle satisfait directement les besoins biologiques, ce qui lui permet d'intervenir comme lien entre l'homme et la nature, mais aussi entre les hommes, comme cela devient manifeste par les réseaux d'information. En ce sens, la technique a vocation à remplacer «efficacement et complètement » le langage et la religion, à condition cependant que la technique soit l'alliance d'une métrologie humaine et d'une énergétique humaine, c'est-à-dire qu'elle instaure une réciprocité réelle entre l'homme et ses concrétisations objectives.

Simondon conclut, comme pour la théorie des phases qu'il propose dans $\mathrm{Du}$ mode d'existence des objets techniques, que «seule une pensée philosophique est commune au progrès du langage, au progrès de la religion, au progrès de la technique ; la réflexivité de la pensée est la forme consciente de résonance interne de l'ensemble formé par l'homme et la concrétisation objective ; c'est cette pensée qui assure la continuité entre les phases successives de progrès, et c'est elle seule qui peut maintenir la préoccupation de totalité, et faire ainsi que la décentration de l'homme, parallèle à l'aliénation de la concrétisation objective, ne s'effectue pas ${ }^{26} »$.

\section{La théorie communicationnelle du langage}

Sur quels éléments une théorie " simondonienne » du langage pourrait-elle malgré tout se constituer? Telle serait la question à poser en guise de conclusion provisoire à cette première enquête. Il ne s'agit pas de nier en dernier ressort l'aporie initiale, ni de la redoubler par une tentative de retournement de la critique du langage et de réduction abstraite des tensions internes évoquées, mais plutôt d'ouvrir quelques pistes possibles. Ces pistes ont en commun la critique fondamentale du logocentrisme et de l'anthropocentrisme, à la fois condition et horizon de toute réflexion sur la signification, l'expression et la communication.

Parmi ces pistes, si on laisse de côté l'idée du langage pensé en tant que "système d'individuation » et en tant qu' " objet technique » - ce qui nécessiterait une relecture des deux thèses en mettant entre parenthèses la critique du langage qui vient d'être exposée -, la piste la plus élaborée est celle d'une théorie communicationnelle du langage. 

Il importe alors d'affirmer que la communication n'est pas primitivement ni prioritairement discursive, ni même intrinsèquement linguistique ; comme le montre la théorie de l'individuation, elle existe avant le langage et même avant la vie, au sein de systèmes métastables capables d'amplification structurante. En son sens le plus général, la communication est donc la résolution par une information d'une disparation, d'une incompatibilité au sein d'un système à l'état métastable, au niveau physique, biologique et psycho-social. Pour le vivant humain, avant de prendre la forme d'un système de signes complexes, la communication est déjà " signification » au niveau biologique primaire et «langage » au niveau éthologique intermédiaire. C'est pourquoi la relation de l'organisme au milieu, tout comme les motivations instinctives dans la perception des signaux des autres êtres persistent et orientent le langage humain complexe : en suscitant l'action, en synchronisant les conduites, en servant de critère pour reconnaître, intégrer et exclure les autres êtres ${ }^{29}$. Au-delà des niveaux biologique et éthologique, le niveau psychique apporte non seulement un stockage et un traitement de l'information différent des niveaux précédents, mais il apporte aux espèces "à intelligence ", et notamment aux espèces sociales comme l'espèce humaine, une tendance vers l'universel qui permet la formalisation des unités sémantiques dans des systèmes symboliques capables de transcender les individus et les cultures. C'est par cette formalisation qu'une communication avec les machines est possible sans que le « code commun » établi coupe toute communication avec les niveaux biologique et éthologique de la relation de l'être sujet au milieu. Multidimensionnelle, polyphasée, relationnelle et irréductible au langage, cette théorie générale de la communication serait la condition fondamentale d'une culture universelle en mesure d'incorporer tout ce qu'est l'homme, tout ce qu'il produit, mais aussi tout le vivant dans lequel il est compris.

S'il fallait finalement ramasser cet état des lieux en une seule proposition, on pourrait dire que Simondon n'est pas un penseur du langage, mais un penseur des conditions d'émergence du langage à travers les phases et les niveaux de la relation de l'individu au milieu au cours de son évolution. Le sens du langage n'est plus alors celui de la vérité comme adéquation de la pensée et du monde, ni l'expression logocentrique et anthropocentrique d'un humanisme abstrait et partiel, mais la résolution de la problématique vitale du sujet à travers la communication universelle. 


\section{BIBLIOGRAPHIE}

Aristote, Organon I-V, trad. fr.J. Tricot, Paris, Vrin, 2000.

Bachelard Gaston, La philosophie du non, Paris, Puf, 2002.

Barthélémy Jean-Hugues, Simondon, Paris, Les Belles Lettres, 2014.

Bergson Henri, «Introduction à la métaphysique », in La pensée et le mouvant, Paris, Puf, 1999.

Bontems Vincent, «Bachelard et Lupasco. Logiques, dialectiques et mécanique quantique. », in Symbolon, $\mathrm{n}^{\circ}$ 8, « Bachelard : art, littérature, science », Wunenburger J.-J. et Buse Ionel (éd.), Université de Lyon et Universitatea din Craiovia, 2012.

Duhem Ludovic, Compte-rendu en ligne du Simondon de Jean-Hugues Barthélémy (Belles Lettres, 2014), site du CIDES, http://www.mshparisnord.fr/cides/index.php/comptes-rendusdouvrages.html

Platon, Le Sophiste, trad. N. Cordero, Paris, GF, 1993

Ruyer Raymond, « Les limites du progrès humain », in Revue de métaphysique et de morale, octobredécembre $1958, \mathrm{n}^{\circ} 4$, p. 412-423.

Simondon Gilbert, L'individuation à la lumière des notions de forme et d'information, Grenoble, Millon, 2005.

Simondon Gilbert, Du mode d'existence des objets techniques, Paris, Aubier, 1989, [1958].

Simondon Gilbert, L'invention dans les techniques. Cours et conférences, Paris, Seuil, 2005.

Simondon Gilbert, Communication et information, Chatou, La Transparence, 2010.

Simondon Gilbert, Cours sur la perception (1964-1965), Paris, Puf, 2013.

Simondon Gilbert, Imagination et invention (1965-1966), Paris, Puf, 2014.

Simondon Gilbert, « Naissance de la technologie », in Sur la technique, Paris, Puf, 2014.

Simondon Gilbert, « Les limites du progrès humain », in Sur la technique, Paris, Puf, 2014.

\section{NOTES}

1. Gilbert Simondon, L'individuation à la lumière des notions de forme et d'information, Grenoble, Éditions Jérôme Millon, 2005, p. 23. Noté ILFI.

2. ILFI, p. 24.

3. Une critique du concept, dans sa relation à l'idée et à l'intuition, est également proposée par Simondon dans sa Thèse complémentaire. Cf. Gilbert Simondon, Du mode d'existence des objets techniques, Paris, Aubier, 1989 [1958], p. 235 sq. Noté MEOT.

4. ILFI, note 7, p. 30.

5. Id.

6. Henri Bergson, «Introduction à la métaphysique », in La Pensée et le mouvant, Paris, Puf, 1999, p. 188.

7. ILFI, p. 34.

8. ILFI, p. 36. L'idée de «pluraliser la logique » est sans doute formulée par Simondon à la suite de Bachelard, lequel affirme dans La philosophie du non que, suite aux découvertes de la science 
contemporaine, «il faudra donc bien modifier en quelque manière le jeu des valeurs logiques, bref, il est nécessaire de déterminer autant de logiques qu'il y a de types d'objets quelconques » (Gaston Bachelard, La Philosophie du non, Paris, Puf, p. 111). Voir également l'excellent article de Vincent Bontems où les conséquences de la physique quantique en logique sont étudiées chez Bachelard et Lupasco mais aussi chez Simondon, «Bachelard et Lupasco. Logiques, dialectiques et mécanique quantique ", in Symbolon, $\mathrm{n}^{\circ}$ 8, «Bachelard : art, littérature, science », Wunenburger J.-J. et Buse Ionel (coord.), Lyon, Université de Lyon III, 2012, p. 251-266.

9. ILFI, p. 36.

10. Barthélémy donne à cette formule deux sens, qui sont en même temps deux "règles » fondamentales de l'unité de l'œuvre de Simondon: «penser au milieu et pas seulement à l'individu » et «penser au milieu en partant du centre de l'être ». Voir Jean-Hugues Barthélémy, Simondon, Paris, Les Belles Lettres, 2014.

Voir également mon compte-rendu de cet ouvrage sur le site du CIDES :

http://www.mshparisnord.fr/cides/index.php/comptes-rendus-douvrages.html

11. Simondon, ILFI, p. 307.

12. Id.

13. Simondon, MEOT, p. 97.

14. Simondon, MEOT, p. 97-98.

15. Cette opposition entre image et langage peut paraitre problématique, en trois sens au moins. Dans un premier sens, elle semble incohérente avec une philosophie qui cherche systématiquement à dépasser les oppositions conceptuelles héritées de l'histoire de la métaphysique, ce que Simondon a pourtant clairement établi comme condition de toute connaissance. Dans un deuxième sens, cette opposition entre image et langage semble abstraite, dans la mesure où l'image paraît complètement séparée de toute détermination du langage dans sa formation et dans son interprétation. Dans un troisième sens, elle semble réductrice, l'image opposée au langage se confondant finalement avec le schéma qui en exprimerait comme l'essence ou la véritable universalité. Si ces trois objections peuvent paraître légitimes, elles ne sont pas totalement fondées : d'abord parce que l'opposition entre image et langage peut se comprendre génétiquement comme un déphasage qui structure l'évolution de l'encyclopédisme, la phase technologique étant la résolution dans la théorie de l'information de l'incompatibilité du langage et de l'image pour exprimer la réalité technique universellement. Ensuite, parce que l'image est effectivement liée au langage et même à l'oralité, ce qui nécessite un effort considérable de formalisation pour détacher l'image du langage, effort accompli depuis la Renaissance et qui reste encore inachevé au xxe siècle dans la cybernétique ; mais il faut ajouter que l'image est plus primitive que toute signification langagière et que toute culture instituée, car elle correspond au niveau pré-subjectif et pré-objectif de la relation de l'individu au milieu, celui du couple sensations-tropismes avant que tout élément géométrique n'intéresse la perception (voir MEOT, p. 192) ; enfin, la pluralité des images n'est pas explicitement niée ni contestée par Simondon, le schéma apparaît seulement comme l'image la plus directe, la moins chargée de significations culturelles constituées, c'est-à-dire la plus adéquate à exprimer l'universalité complète de l'encyclopédisme technologique. Il reste à préciser que l'image seule ne peut suffire, même sous la forme d'un schéma rationnel, pour accomplir la réforme culturelle que nécessite l'encyclopédisme technologique; elle doit être accompagnée par une éducation technique qui nécessite une mise en situation opératoire du sujet et une formulation de la pensée qui passe aussi, mais non pas prioritairement ni exclusivement, par le langage.

Il faudrait une étude spécifique pour élucider la relation entre image et langage, ce qu'il est impossible de fournir ici. Quelques éléments se trouvent dans le cours Imagination et invention (voir Gilbert Simondon, Imagination et invention (1965-1966), Paris, Puf, 2014).

16. Simondon, MEOT, p. 98.

17. Ibid., p. 100. 
18. Voir « Allagmatique », texte faisant partie des suppléments d'ILFI, p. 559-561.

19. Simondon, MEOT, p. 180.

20. Ibid., p. 183.

21. Ibid., p. 195.

22. Gilbert Simondon, «Les limites du progrès humain », in Sur la technique, Paris, Puf, 2014. Noté LPH.

23. Raymond Ruyer, "Les limites du progrès humain ", in Revue de métaphysique et de morale, octobre-décembre 1958, $\mathrm{n}^{\circ}$ 4, p. 412-423 (les considérations sur le « langage » se trouvent p. 421).

24. Malgré ce recours, le langage ne peut pas être un paradigme pour penser l'évolution technique. Simondon l'affirme dans deux textes postérieurs, en critiquant directement la méthode structuraliste. Cf. Gilbert Simondon, «L'invention et le développement des techniques (1968-1969) », in L'invention dans les techniques. Cours et conférences, Paris, Seuil, 2005, p. 85, et Gilbert Simondon, «Naissance de la technologie », in Sur la technique, Paris, Puf, 2014, p. 169. La place manque ici pour discuter de cette question.

25. Simondon, $L P H$, p. 272-273.

26. Ibid., p. 278.

27. Cf. Gilbert Simondon, Imagination et invention (1965-1966), Paris, Puf, 2014 ; Gilbert Simondon, Cours sur la perception (1964-1965), Paris, Puf., 2013 ; Gilbert Simondon, Communication et information, Chatou, La Transparence, 2010.

28. Gilbert Simondon, Cours sur la perception, op. cit., p. 112.

29. Cf. Gilbert Simondon, Communication et information, op. cit., p. 65-66.

\section{RÉSUMÉS}

La question du langage dans la pensée de Simondon est une question aporétique. Alors que toutes les conditions semblent réunies pour qu'une théorie originale et cohérente soit élaborée, il n'existe dans son œuvre que des réflexions éparses qui ne donnent pas lieu à une théorie mais plutôt à une critique non systématisée du langage. Deux hypothèses peuvent expliquer cette «absence » de théorie du langage : selon la première, Simondon n'avait pas besoin du langage pour repenser les conditions de la pensée, du sens et de la vérité, la théorie de l'individuation proposant une ontologie, une logique et une épistémologie pour lesquelles le langage n'est ni premier ni central ; selon la deuxième, Simondon a posé autrement la question du langage, sous la forme d' une théorie de la communication qui se fonde sur le rapport entre information et signification, lequel dépasse et englobe la question du langage. À partir de ces deux hypothèses, il est possible de poser que Simondon a cherché à sortir la philosophie du logocentrisme et de l' anthropocentrisme réductionniste qui le soutient, ce qui implique une relativisation fondamentale du langage. Cet article propose en ce sens de faire un premier « état des lieux» de la question du langage dans l'œuvre de Simondon à travers l'étude :

1. de sa critique du langage compris comme condition de la connaissance et de la pensée ;

2. des tensions internes au rapport entre technique et langage chez Simondon;

3. des éléments qu'il pose pour une théorie communicationnelle du langage. 
INDEX

Mots-clés : communication, encyclopédisme, image, individuation, langage, logique, signification, sujet, technique, universel

\section{AUTEUR}

\section{LUDOVIC DUHEM}

Philosophe et artiste, Responsable de recherche à l'École Supérieure d'Art et de Design d'Orléans et de Valenciennes, Membre du CIDES et du comité mésologiques.fr ; ludovicduhem@orange.fr 